\title{
Comparison Between New Confidense HD Coloring Carto Mapping Module and New Rhythmia Mapping System for Scar Related Atrial Tachycardia Treatment.
}

\author{
Nicolas Vidal ${ }^{1}$, ANTOINE DA COSTA ${ }^{2}$, Cecile Romeyer ${ }^{3}$, Jean Baptiste Guichard ${ }^{1}$, \\ Karim Benali ${ }^{4}$, Cedric Yvorel $^{4}$, and Karl Isaaz ${ }^{5}$ \\ ${ }^{1}$ Université Jean Monnet Saint-Etienne \\ ${ }^{2}$ UNIVERSITY OF SAINT ETIENNE \\ ${ }^{3}$ University of Saint Etienne \\ ${ }^{4}$ Jean Monnet University Saint-Etienne University Institute of Technology \\ ${ }^{5}$ Saint-Etienne University Hospital Bellevue Site
}

July 5,2021

\begin{abstract}
Background. Scar-related atrial tachycardias (ATs) are very challenging and no comparative studies have been conducted between the up-to-date systems, namely the new Confidense HD coloring mapping module (HD) and the new Rhythmia (RM) mapping system. Objectives. Our study sought to compare both strategies in terms of: (1) diagnosis and immediate treatment success: (2) procedure parameters in AT presenting patients. Methods and Results. Overall, 38 patients (68 \pm 9 years old) were randomized $(\mathrm{HD}=20, \mathrm{RM}=18)$. The AT mechanisms did not differ between the groups: macro/small loop reentrant mechanisms were more common in both groups $(12 / 20$ [60\%] versus $10 / 18$ [56\%], $\mathrm{P}=0.9)$, with the remaining diagnosed as multiloop $(5 / 20$ [25\%] versus 4/18 [22\%]; $\mathrm{p}=0.9)$; focal $(1 / 20$ [5\%] versus $2 / 18$ [11\%], $\mathrm{P}=0.9)$, and localized reentrant $(2 / 20$ [10\%] versus $2 / 18$ [11\%]). The procedure parameters were as follows: tachycardia cycle length ( $275 \pm 30$ vs. $280 \pm 50 ; \mathrm{p}=0.7)$; number of activation maps per patient $(2 \pm 1$ vs $2 \pm 1 ; \mathrm{p}=0.9)$; procedure time ( $138 \pm 90$ vs. $139 \pm 90 \mathrm{~min} ; \mathrm{p}=0.98)$; X-ray time exposure ( $20 \pm 11$ vs. $24 \pm 18 \mathrm{~min} ; \mathrm{p}=0.42)$; RF application time ( $39 \pm 36$ vs. $25 \pm 28 \mathrm{~min} ; \mathrm{p}=0.21)$; and mean number of circuits per patient ( $1.8 \pm 1$ vs. $1.6 \pm 0.9 ; 0.6)$. Acute success was obtained in $15 / 20 \mathrm{pts}(75 \%)$ of the HD group and $14 / 18(78 \%)$ of the RM group (p=0.9). The procedure failed in 2 patients with the HD coloring and they were converted to the RM group without success. No crossover was carried out in the RM group. Conclusions. This randomized study demonstrates that HD coloring and Rhythmia systems exhibit a similar efficacy in achieving acute AT termination.
\end{abstract}

\section{Introduction}

Although circumferential pulmonary vein ablation (CPVA) has proven effective in eliminating atrial fibrillation (AFib), this procedure may result in the development of organized scar-related atrial tachycardias $(\mathrm{ATs})^{1-3}$ that require repeat ablation procedures ${ }^{1-3}$. Thus, scar-related ATs are very challenging and likely to occur more frequently either post-AFib ablation, post-surgical incisional, or spontaneous in the presence of degenerative atrial diseases ${ }^{1,3,4}$. The mechanism of these arrhythmias usually consists of reentry involving pre-existing or iatrogenic ablation-related scar tissues. These circuits are often challenging to map because of significant scar with fractionated multicomponent electrograms, thereby limiting accurate time annotation ${ }^{1-3}$. ECG-based diagnosis, electroanatomical mapping, entrainment mapping, substrate imaging, and detailed knowledge of previously-created lesions are all employed for the circuit diagnosis (or the original site in case of focal propagation $)^{3-6}$. The current gold standard approach to AT mapping relies on the annotation of the local activation time (LAT) of each intracardiac electrogram collected within a prespecified 
window of interest (WOI) based on the tachycardia cycle length (TCL) ${ }^{2}$. Nevertheless, these techniques are time-consuming and challenging, possible yielding confusing results ${ }^{1,3-8}$. Recently, multipolar catheters were developed especially for multi-electrogram acquisition (PentaRay Nav $\AA$, Biosense Webster; Orion $\AA$, Boston Scientific) ${ }^{9-13}$ with significant advances in mapping resolution. The newly released electroanatomical mapping (EAM) systems enable fast and fully automatic acquisition with accurate automatic annotation of multiple electrograms upon activation mapping. These systems permit better definition of the circuits and accurate identification of the targets that need to be abolished. Several options have been proposed to improve the diagnosis mechanism and treatment efficacy of scar-related $\mathrm{AT}^{9-14}$. Multielectrode acquisition was first developed to ameliorate resolution mapping, which is influenced by electrode size and interelectrode spacing. Smaller electrodes with closer interelectrode spacing may improve mapping resolution, particularly in case of scar ${ }^{9-14}$. Ideally, complex circuits require ablation of a critical isthmus (CI), which is sometimes common to several reentry loops, so as to obtain immediate and definitive AT interruption. Using the currently available mapping tools, CI can prove to be difficult or even impossible to visualize in severely scarred areas ${ }^{3,4,9-14}$. The PentaRay multielectrode mapping catheter is a 20-pole steerable mapping catheter arranged in five soft radiating spines covering a diameter of $3.5 \mathrm{~cm}$ (PentaRay, Biosense Webster; Diamond Bar; inter-electrode spacing 2-6-2mm-multielectrode-mapping) whose ability to improve AT's diagnostic mechanisms has been validated ${ }^{10-12}$. The new Rhythmia (Boston Scientific) mapping system uses a 64-pole basket mapping catheter (IntellaMap Orion; Boston Scientific), which incorporates small unidirectional electrodes $\left(0.4 \mathrm{~mm} ; 2.5 \mathrm{~mm}\right.$ spacing) to suppress noise and far-field signals ${ }^{13,14}$. Multielectrode acquisition and highly reliable automatic annotation yield maps with a previously-unattained resolution ${ }^{10-14}$. As recently emphasized, the closer interelectrode spacing (center to center $2.5 \mathrm{~mm}$ for Orion versus $3 \mathrm{~mm}$ for PentaRay and $4.75 \mathrm{~mm}$ for standard 3.5-mm tip mapping catheters) enables recording of a higher bipolar voltage amplitude ${ }^{10-14}$. Additionally, high-density automated mapping of atrial arrhythmias has gained increasing interest, as it allows for fast and high-resolution activation mapping, thereby reducing both the mapping and overall procedure times ${ }^{15-18}$. The Confidense TM mapping module (Carto 3 v. 4, Biosense Webster) as well as the new Rhythmia (Boston Scientific) mapping system were developed to improve accurate diagnosis of AT circuits in severely scarred atria, and reliable CI localization, which consistently shows much lower electrogram amplitude and significantly slower conduction velocities (CV) than the surrounding tissues and other parts of the circuit. Once identified, ablation of isthmuses of slow conduction may rapidly result in a high success rate ${ }^{15-18}$. The new features of the 3D EAM system CARTO 3 Version 6.0, high-definition (HD) coloring (Biosense Webster, Diamond Bar, CA) may also improve the high-quality display of the EAM; in addition to the conventional early-meets-late (EML), this system highlights areas of potential conduction block, called extended early-meets-late (EEML), thereby enabling better interpretation of the LAT and propagation map ${ }^{19}$. In this specific area of development with different approaches of mapping catheters and 3D EAM softwares, no true comparison has so far been performed between both strategies. Accordingly, our prospective randomized study sought to compare both strategies in terms of: (1) diagnosis and immediate treatment success; (2) procedure parameters in patients with scar-related ATs.

\section{Methods}

According to institutional guidelines, all patients were informed of and provided written consent for the study and its invasive procedures. The study was approved by the Institutional Committee on Human Research at the authors' institution. Antiarrhythmic drugs were discontinued at least five half-lives before the procedure, except for amiodarone, which was interrupted 1 month before the procedure if possible. The data supporting the findings of the study were made available from the corresponding author upon reasonable request. The patients were recruited among those referred to our center for paroxysmal/persistent AT ablation. Subjects were excluded from participation if the documented ECG was consistent with typical cavotricuspid isthmusdependent flutter. To guide ablation, the patients were block randomly assigned, using an unblinded 1:1 mapping design, to either HD coloring mapping or Rhythmia mapping. The randomization was performed via a sealed envelope.

Patient Selection. Consecutive patients were included if they underwent radiofrequency ablation for permanent AT, irrespective of its etiology, or if AT ablation had occurred following an initial AF ablation index 
procedure. Between January 2019 and September 2019, 60 patients were recruited, with 22 excluded due to premature AT termination $(\mathrm{n}=13)$, AFib the day of the procedure $(\mathrm{n}=4)$, left atrial appendage thrombus $(n=4)$, or other diagnosis (intra-nodal reentry). Overall, 20 patients were preliminary included in the PentaRay-HD coloring group and 18 patients in the Orion-Rhythmia group. All mapping procedures were performed using either a PentaRay catheter or Orion catheter. Randomization between both groups was performed at least 1 week prior to the procedure. While industry support was offered to assist with the CARTO or Rhythmia operating systems, the industrials also provided assistance in interpreting the activation map to which each patient was randomized. The patients displayed conventional ablation indications, such as symptomatic AT or failed attempt with antiarrhythmic drugs to maintain sinus rhythm. The exclusion criteria were hyperthyroidism, left atrial (LA) thrombus, decompensated heart failure, stroke, myocardial infarction, or gastrointestinal bleeding within 4 weeks prior to the intervention, as well as life expectancy $<6$ months. Written informed consent was obtained from all patients.

AT Catheter Ablation. The procedures were performed under vitamin K antagonist continuation (with a target international normalized ratio of 2.0-3.0), uninterrupted direct oral anticoagulant (dabigatran, rivaroxaban, or apixaban) under conscious sedation, or general anesthesia in a fasting state. All catheters were advanced via the femoral vein. A decapolar catheter was placed into the coronary sinus (CS), with a suitable CS reference selected. Burst pacing down to $200 \mathrm{~ms}$ from different CS poles was applied to induce $\mathrm{AT}$ if the patient was in sinus rhythm at the start. Intravenous heparin was administered immediately after the insertion of the femoral sheaths and continuously given to maintain an activated clotting time of 300$350 \mathrm{~s}$ throughout the procedure. A 3.5-mm open-irrigated magnetic ablation catheter (Navistar Thermocool RMT, Biosense Webster Inc.) or contact force sensing catheter (Smart Touch, Biosense Webster Inc.) was then advanced through an SL1 sheath (St. Jude Medical, Inc.) into the left atrium (LA) in both groups. Subsequent heparin boluses were administered every 20-30 minutes if needed via a heparinized Swartz SL0 sheath (Saint Jude Medical).

PentaRay and HD Coloring Confidence Mapping. Previous ablation history and CS activation pattern in AT were used to decide on the first chamber to map. The CARTO 3v4 CONFIDENSE (Biosense, Inc) was employed for mapping with a PentaRay Nav (Biosense, Inc). A point density to color the entire geometry with a color threshold of $5 \mathrm{~mm}$ was targeted. The criteria for including points on the map using CONFIDENSE Continuous Mapping included the cycle length stability within a 5\% range of the TCL, electrode position stability within $2 \mathrm{~mm}$, LAT stability filter at 3 milliseconds, and tissue proximity to the endocardial surface. We performed CS catheterization using a decapolar diagnostic catheter (CS catheter F-type, 2-8-2 $\mathrm{mm}$ spacing, Biosense Webster). After LA thrombus exclusion using a guided double transseptal puncture, a multipolar catheter (PentaRay, 2-6-2 $\mathrm{mm}$ interelectrode spacing, $1 \mathrm{~mm}$ electrodes) was advanced within a sheath into the LA (St. Jude Medical, Inc.). The Carto 3 system enables real-time Advanced Catheter Location TM and visualization of both ablation and mapping catheters (PentaRay). The catheter location display is identical to that of the fluoroscopic view. The Carto 3 system combines electromagnetic technology (as in the Carto XP system; Biosense Webster, CA, USA) with new advanced catheter location technology that permits visualization of multiple catheters without fluoroscopy. Briefly, Advanced Catheter Location is an impedance-based catheter localization system that enables precise cardiac mapping and navigation with multiple electrodes. Six electrode patches are attached to the body surface and constantly monitor the current emitted at a frequency unique to each individual catheter electrode. Each electrode patch is equipped with a magnetic sensor, enabling 3D localization. Currents detected at the patches relate the electrodes' 3D positioning inside the human body. The Carto 3 system's ability to visualize the manipulation and placement of multielectrode mapping catheters in each part of the LA may further reduce fluoroscopy time. This new system improves catheter stability during ablation and eases identification of each catheter electrode-pair position. Visualization of catheters and electrode pairs is provided via a real-time, on-screen display of a geometrically reliable icon representing the PentaRay catheter along with the electrodes' positions. LA reconstruction was obtained using a fast anatomical map algorithm. Based on this volume sampling, a surface reconstruction was built in accordance with the set resolution level to prevent LA volume overestimation owing to respiratory movements. Once the map was completed, a 3D computed tomography scan was in- 
tegrated to optimize the LA reconstruction. During AT, based on analysis of the surface ECG atrial wave morphology and activation sequence on the CS catheter, the applied strategy consisted of activation mapping using the PentaRay with the electroanatomical system in either the right atrium, LA or both. Sites including fractionated electrograms, double potentials ([?]50 ms), and very low amplitude electrograms $(<0.05 \mathrm{mV})$ as well as atrial scars were systematically labelled on the map. Conventional activation mapping involves measuring the LAT of each electrogram. This denotes the numerical difference in timing between a component of a sampled electrogram and stable reference signal, the values of which are plotted on the 3D map according to a rainbow-colored bar that spans the interval of the mapped tachycardia. Annotation of LAT requires a timing WOI in order to ensure that electrograms from the same beat are compared. A WOI is defined by specifying a timing interval both before and after the reference point. The position of the reference point in relation to the window is arbitrary. The color red is employed to represent the earliest LAT measurement within the specified window, and purple is used to represent the latest. In a reentrant tachycardia, the "early" and "late" concept is a misnomer because any given activation area will have other sites that are activated before or after that location, with the red area of the earliest activation adjacent to the purple area of the latest (i.e., early-meets-late). All acquired points were both automatically and manually checked and annotated. Care was taken to annotate the LAT for each acquired point according to the positive deflection peak of the bipolar signals on the multipolar catheter. HD Coloring (CARTO 3V6; Biosense Webster, CA) is a recently released software that provides a higher quality display of the electroanatomical map due to a higher density of points. It enables the user to set up two ranges of early-meets-late: an upper range (the possible reentry zone) and lower range for a possible block mechanism-EEML. However, the optimal cut-off ranges have not yet been defined. The CARTO 3v4 CONFIDENSE module offers several features designed to enhance LAT mapping, including wavefront annotation, as well as an automated algorithm that assigns timing annotation at the maximal negative distal unipolar derivative within the time window spanned by the corresponding bipolar electrogram. In addition, there is an algorithm enabling an automated WOI to recompute, which allows for the WOI and subsequent LAT of each projected point to be recalculated at any time upon map acquisition. There is also a map consistency filter, which determines the consistency of each measured LAT point relative to its neighboring points, while highlighting outlier points with LATs very different to their surrounding neighbors for review, deletion, or re-annotation, as necessary. In this arm of the study, during the geometry and electroanatomic map acquisition, the LAT and bipolar voltage maps were displayed. The WOI was set from the start, spanning between $90 \%$ and $100 \%$ of the TCL and either around the reference signal or $P$ wave surface ${ }^{20}$. Operators were able to adjust the WOI as required using the automated recalculation function for the complete electroanatomic map. Automated annotation of activation timing was assigned using the CONFIDENSE Wavefront algorithm in all cases. In the absence of a sharp negative slope $>0.03 \mathrm{mV}$ in the unipolar signal, a grey square was projected, as the system could not automatically assign the LAT. Once all points had been collected, the Map Consistency filter was used as required. The static LAT map was then visualized as a color propagation map. Additional manual LAT reannotation or deletion was permitted at sites where propagation remained unclear on the map. In a reentrant circuit, an early-meets-late algorithm could be applied in order to interpolate between early and late sights on the map considered reentrant.

Orion and Rhythmia Ultrahigh Resolution EAM. Detailed EAM of the right atrium (RA) and LA was performed using bidirectional flexion with the basket in variable deployment degrees (diameter ranging $3-22 \mathrm{~mm}$ ). The location of each of the 64 electrodes was displayed on the mapping system using a combination of the magnetic sensor (located in the distal catheter region) and impedance measurement of each basket electrode. Two reference electrograms (EGM; one main $[R]$ and one additional $[\Delta R]$ ) were chosen on the decapolar catheter. Cardiac beats were automatically selected for inclusion into the map based on the cycle length (CL) stability, stable relative timing of two reference EGMs, electrode location stability, and respiratory gating. The WOI was automatically set by the system at the CL value and centered on the main reference EGM. For LAT annotation of each acquired bipolar EGM, the system combined unipolar (maximum negative $\mathrm{d} V / \mathrm{d} t$ ) and bipolar (maximum amplitude) EGMs. For fragmented or multiple potential EGMs, the system took into account the timing within the surrounding area in order to select the potential to apply for the timing annotation. In the event of a statistical incoherence between neighboring points 
within an area, no color code was displayed (the area is left gray). Scar setting could be finely tuned with the confidence mask tool (points in the immediate surrounding area with EGM bipolar amplitude below the confidence mask have no color code and are displayed in gray). The chamber surface geometry was generated using the location of the outermost electrodes, gated to the respiratory and cardiac cycles, while being continuously updated during mapping. Surface EGM selection was based on the projection distance, which could be set between 1 and $5 \mathrm{~mm}$, with only EGMs recorded within the projection distance from the surface geometry displayed. Activation and voltage maps were studied without changing the system's automatic timing annotation. Activation maps, concerning both RA and LA, were studied for each AT. The right or left origin was rapidly diagnosed based on the CL coverage degree or by localizing the earliest activation site upon the simultaneous display of both cavities in the event of focal arrhythmias. Wavefront propagation was visualized following a $10 \mathrm{~ms}$ activation window, which was slowly advanced along the timescale. Contrarily to previously available mapping systems, Rhythmia could display a circular WOI , thereby eliminating the artificial early and late LAT classification, deemed inappropriate for reentries. The isthmus region was chosen based solely on propagation (sites of narrowing or slowing of the wavefront) for the Rhythmia system. Due to its much higher resolution degree, mapping has been postulated to provide sufficient accuracy to prevent misinterpretation. The system calculated the median AT cycle length over 10 seconds and set the WOI (WOI) duration to $100 \%$ of the observed CL, centered on the timing reference electrode. Beats were automatically and continuously collected. They were accepted for display on the map only after having met a beat acceptance criterion series: stable CL within $10 \mathrm{~ms}$ of the target AT CL; relative timing deviation of $5 \mathrm{~ms}$ between two CS reference electrodes; respiration gating in the expiration phase with a threshold set at $50 \%$ of the peak-peak amplitude; stable catheter location within $1 \mathrm{~mm}$; stability of the mapping catheter signal morphology of at least $75 \%$ from beat to beat. Only EGMs within $2 \mathrm{~mm}$ of the surface geometry were displayed and applied for map computation. When available, we used the Lumipoint module in order to help the LAT interpretation map ${ }^{5-18}$. This module includes several tools such as the automatic sensing of fractioned or double potentials, helping to localize potential isthmus, conduction block and/or gap. Moreover, to assist the detection of critical isthmus (CI), the Lumipoint module displays the amount of surface area activating at specific times during the full tachycardia cycle length allowing a full chamber activation view. This tool allows to select the period when the smallest amount atrial surface is active by unit of time (skyline's valley) and thus to select one or more potential isthmus in the LAT map ${ }^{5-18}$.

Bipolar Voltage Map Assessment for Both Systems. Following pulmonary vein (PV) exclusion, the total LA surface was calculated by means of the surface measurement tool for all LA maps. We additionally measured the extent of the low voltage areas (for bipolar cutoffs of $0.5 \mathrm{mV}$ and $0.05 \mathrm{mV}$, in line with previously published and accepted data), in addition to that of the LA surface below the selected confidence mask for each map.

AT Diagnosis. Entrainment mapping was employed only when the diagnosis had not been made, in an effort to facilitate the cartography. As proposed since 2001, AT were classified in macroreentries and focal arrhythmias. Macroreentry was defined as AT propagating around a central obstacle (scar or anatomical structure, such as a valve or PV ostia), with a [?]90\% CL coverage within the chamber of origin. When there was no clearly distinguishable central obstacle (no central dense scar) or the potentials over a small area covered [?]90\% of the CL, along with a centrifugal activation of the remaining atrial regions, the AT was considered a microreentry, reflecting a mechanism of focal tachycardia. Purely focal ATs were those without CL coverage $(<90 \%)$ and centrifugal chamber activation from the earliest activation site. The AT was considered to be a macro-reentrant in the event it traveled continuously around the mapped atrial chamber (where head met tail), while small loop circuits were contained within a single plane (i.e ., the anterior wall). Both focal/localized reentrant circuits were likely to emanate from a small focus, spreading radially away and often involving low voltage and fractionated EGMs at the break-out site. CI was defined by the narrowest or slowest portion on the circuit, where RF ablation was applied.

Procedure of Ablation. Selecting the ablation target was solely based on circuit and isthmus visualization, without entrainment mapping. For focal AT, the earliest activation site was targeted. Acute success was defined as AT termination with sinus rhythm resumption or its change to another stable AT upon ablation, 
without intervening atrial extrasystoles. An ablation-induced transformation to another stable AT was presumed if there was an abrupt and sustained change in CL, intracardiac activation pattern, and surface ECG. Minor $(<10 \%)$ CL prolongation alone, not accompanied by changes in intracardiac activation pattern or surface ECG atrial wave morphology, was not considered to be an acute success. If the AT was unchanged following CI ablation, the procedure was considered a failure, with another target chosen for ablation. The conduction block was assessed across lines during pacing on one side and mapping on the other side in order to differentiate persistent but slowed conduction from a complete line block. The power setting was programmed at 25 to $40 \mathrm{~W}$ with an optimized catheter-tissue contact if available (between 10 and $40 \mathrm{~g}$ ). $\mathrm{RF}$ was administered in a point-by-point manner, without dragging. Additional ablation was performed for PV disconnection, as necessary. Additional programmed stimulation was systematically performed. In the event of supplemental AT in a given patient, remapping and ablation were performed up to sinus rhythm resumption. Circumferential PV isolation was carried out when necessary using a 3D mapping system (Carto 3, Biosense Webster Inc.), in conjunction with the LA integrated computed tomography image and real-time fluoroscopy. The radiofrequency generator (Stockert, Biosense Webster Inc.) was set to temperature-controlled radiofrequency delivery with a target temperature of $42^{\circ} \mathrm{C}$ and nominal power limits of $40 \mathrm{~W}$ (flow $30 \mathrm{~mL} / \mathrm{min}$ ) and $30 \mathrm{~W}$ (flow $17 \mathrm{~mL} / \mathrm{min}$ ) at the posterior LA wall. Electrical isolation of all PVs defined as a bidirectional conduction block was proven using the multi-electrode catheter.

Entrainment. In this study protocol, operators were free to use entrainment in both groups if necessary to enable a fair comparison of the two mapping approaches. Thus, if an operator was confident of the AT diagnosis and ablation strategy based on the 3D map, no entrainment was performed. Entrainment maneuvers were permitted if an operator had some diagnostic uncertainty concerning diagnosis validation prior to ablation. For instance, if the mapping approach suggested peri-mitral macro-reentry, entrainment from the CS proximal and CS distal would have been permissible.

Crossover to the Other Mapping Arm. Crossover was carried out if the scar-related diagnosis or treatment had failed, with another procedure scheduled using the other system.

Follow-Up. All patients were monitored in the hospital for [?]12 hours. On the day following the procedure, a 12-lead surface ECG was acquired for normal sinus rhythm confirmation. Previous antiarrhythmic drugs were generally stopped following ablation. Oral anticoagulation was continued after the procedure. After hospital discharge, all patients were followed up in an outpatient clinic at 3,6 and 12 months. At each visit, patients were questioned as for symptom (NYHA and EHRA class) and documented arrhythmia recurrences, with current medication assessed. Additionally, 12 leads ECG, 72-hours Holter recordings and TTE were performed at 3,6 , and 12 months after the last procedure. A documented AF or left AT episode lasting $>30$ s outside a blanking period of 3 months after the index or repeat procedure were considered recurrent $\mathrm{AF} / \mathrm{AT}$.

Study Endpoints. Clinical and procedural data were analyzed for each group. The primary endpoint of this observational study was acute procedural success, defined as successful non-inducible AT ablation with sinus rhythm resumption. The first ablation set encompassed all the ablation lesions delivered to target the AT based on the studied activation map. For instance, if the mapping approach suggested LA roof dependency, the first ablation set would have been a roof line(s), and AT termination after this ablation set alone would achieve the primary endpoint. If AT1 changed to AT2 with ablation, defined as a sustained change in CS activation or CL, the primary endpoint was not considered to have been achieved, with a new cartography performed until the sinus rhythm was restored, and so on. All procedures were performed by two experienced electrophysiologists (ADC and CR). An ablation-induced transformation into another AT was presumed in the event of a sustained change in CL ([?]20ms), intracardiac activation pattern (on the CS catheter), or surface ECG. The endpoint was likewise achieved when an isthmus block was demonstrated in patients who benefited from a mitral isthmus line, roof line, or both. Cases wherein the tachycardia (1) either terminated into sinus rhythm or (2) degenerated into AF prior to mapping was completed were excluded from analysis. This study sought to assess the diagnostic efficacy in both an acute setting and longterm follow-up (12 months). The secondary endpoints were procedure parameters including the procedure 
duration (skin to skin), X-ray exposure, number of cartographies per patient, acquired points per patient, and RF application time per patient. Long-term procedural success was defined as long-term freedom from any AT/AF episodes, irrespective of symptoms, after the index procedure over the 12-month followup without antiarrhythmic drugs. Further tertiary endpoints were procedure-related complications, such as death, atrio-esophageal fistulae, PV stenosis requiring intervention, pericardial tamponade, systemic embolic events, phrenic nerve paralysis, femoral vessel damage requiring surgery, blood transfusion, or hospitalization prolongation.

Statistical Analysis. A prior randomized study demonstrated a diagnostic yield using RM compared with LAT maps of $90 \%$ versus $65 \%^{21}$. Assuming the same effect size, to detect a between-arm difference with $80 \%$ power at the $5 \%$ two-tailed significance level, this required at least 40 patients in each group. A sample size of at least 80 patients was thus targeted ${ }^{21}$. Categorical variables were expressed as percentages, continuous variables as mean+-1 SD for parametric data and median (lower quartile - upper quartile) for non-normal data. Categorical data were analyzed using either a Fisher exact test or $\chi^{2}$ test where appropriate. Unpaired data were analyzed using a Student $t$ test for parametric data and Mann-Whitney $U$ test for non-normal data. A two-sided $P$ value was determined where applicable, with a value of $P[?] 0.05$ considered statistically significant. All analyses were performed using StatView@5.0 (StatView IV, Abacus Concept, Berkeley, CA, USA).

\section{Results}

Patient Population. Overall, 38 patients ( $68 \pm 9$ years old) were randomized $(\mathrm{HD}=20 ; \mathrm{RM}=18)$ with complete mapping and ablation (Table 1). The clinical and echo characteristics did not differ between both groups.

Cardiac AT Characteristics The HD and RM groups displayed similar etiologies (HD versus RM): prior ablation AFib RF ( 8 vs. 11), cardiac surgery (3 vs. 2 ), and naïve procedure (9 vs. 5 ), respectively ( $\mathrm{p}=0.47$ ) (Table 2). AT mechanisms did not differ between the groups: macro/small loop reentrant mechanisms were more common in both groups $(12 / 20[60 \%]$ vs. 10/18 [56\%], $P=0.9)$, with the remaining diagnosed as multiloop (5/20 [25\%] vs. $4 / 18[22 \%]$; $=0.9)$; focal $(1 / 20[5 \%]$ vs. $2 / 18$ [11\%], $P=0.9)$; localized reentrant $(2 / 20[10 \%]$ vs. $2 / 18[11 \%])$. The TCL $(275 \pm 30$ vs. $280 \pm 50 ; \mathrm{p}=0.7)$ was also comparable. AT mechanisms and localizations for each group were described in Table 3 and 4.

Procedure Parameters The number of activation maps per patient ( $2 \pm 1$ vs. $2 \pm 1 ; \mathrm{p}=0.9$ ), procedure time ( $138 \pm 90$ vs. $139 \pm 90 \mathrm{~min} ; \mathrm{p}=0.98)$, X-ray time exposure ( $20 \pm 11$ vs. $24 \pm 18 \mathrm{~min} ; \mathrm{p}=0.42), \mathrm{RF}$ application time ( $39 \pm 36$ vs. $25 \pm 28 \mathrm{~min} ; \mathrm{p}=0.21$ ), and mean number of circuits per patient ( $1.8 \pm 1$ vs. $1.6 \pm 0.9 ; \mathrm{p}=0.6)$ were similar (Table 2). As expected, the number of collected points significantly differed between HD coloring $(6370 \pm 311)$ and $\mathrm{RM}(27870 \pm 2034)$ ( $\mathrm{p}<0.0001)$ but the acquisition time was similar $(13.3 \pm 5.5$ min vs. $13.3 \pm 5.3 \mathrm{~min} ; \mathrm{p}=0.96)$. Acute success was completely obtained in $15 / 20$ patients $(75 \%)$ of the HD group and $14 / 18(78 \%)$ of the RM group ( $\mathrm{p}=0.94)$. In $3 / 20(15 \%)$ vs. $3 / 18(16.6 \%)(\mathrm{P}=0.95)$ patients, the success was incomplete due to ablation-induced transformation to another AT, whereas, despite another cartography, the sinus rhythm restoration was not obtained. One patient from the HD coloring was considered a failure, though the mapping activation was not performed due to a tamponade during transseptal access (existence of an Amplatzer). The complication was resolved using a pericardiocentesis without any consequences and no other complications were observed in the remaining population.

\section{Discussion}

Major Findings. AT mapping can prove challenging owing to significant scar with long, fractionated, and multicomponent EGMs, thereby limiting accurate local time annotation. In these cases, distinction between local ("near-field") and remote ("far-field") signals can be difficult, with LAT often annotated subjectively at the EGM onset or at the highest frequency component, often considered to be local. While mapping accuracy can be improved while using catheters with smaller electrodes and shorter interelectrode spacing, complex EGM annotation remains challenging, only $54.4 \%$ of all low-voltage data points having distinct EGMs to enable accurate activation time. New mapping technologies offer several potential advantages, 
including (1) multielectrode basket catheters with small and closely spaced electrodes, increasing mapping resolution; (2) rapid data acquisition, permitting a sampling density at a magnitude higher than conventional mapping systems; (3) activation time determination using the combination of bipolar and unipolar EGMs, with local timing at the maximum (-) dV/dt of the unipolar EGM; (4) novel algorithms for map coherence determination (in sites with multiple potentials, EGM timings in the surrounding area are employed to determine the most physiological potential for timing); (5) automated data acquisition based on tachycardia CL, EGM, and activation stability. Given this clinical setting, to the best of our knowledge, our study is the first prospective randomized study comparing two new systems incorporating both technologies, namely catheters with smaller multielectrodes and an HD automatized mapping system. Two different approaches have been developed: the Confidense HD coloring mapping module (Carto 3 v. 4, Biosense Webster) and the new Rhythmia (Boston Scientific) mapping system using the up to date systems 3D activation mapping techniques ${ }^{10-18}$. Despite significantly more automatically acquired points with RM system, our preliminary results found that first intent LAT mapping on the HD coloring (CARTO3v4 CONFIDENSE platform) exhibits a similar AT diagnosis termination rate using the PentaRay catheter compared to the combination of the Orion catheter and RM system.

Comparison of Pentaray Multielectrode and Orion Catheter Technologies. Multielectrode catheters (PentaRay, Biosense Webster Inc.) are now widely employed upon AF ablation procedures at different levels during either tachycardia or sinus rhythm for ${ }^{10-14,19}$ : fast anatomical mapping and geometrical creation of the LA shell; PV mapping and confirmation of persistent isolation; voltage mapping to identify reconnected or atrial fibrotic regions; complex fractionated atrial EGM mapping using dedicated software during ongoing AF; activation mapping when AF terminates and converts into AT; voltage mapping during sinus rhythm aimed to identify putative activation channels. The technology has been largely validated, while significantly improving the mapping accuracy in several clinical situations, in comparison with conventional mapping ${ }^{10-14,19}$. Another concept and design created to improve arrhythmia diagnosis and treatment have been developed by Boston Scientific. The construction design of the mini-basket Orion catheter appears to be unique. The electrodes are flat and smaller $\left(0.4 \mathrm{~mm}^{2}\right)$ than those of any other diagnostic catheter that incorporates $1 \mathrm{~mm}$ ring electrodes (i.e., PentaRay or Lasso; Biosense Webster, Diamond Bar, CA). This provides increased mapping resolution. Owing to their exclusive location on the external side of the spline, these electrodes are naturally less influenced by noise and far-field signals. As recently emphasized, the closer interelectrode spacing (center to center $2.5 \mathrm{~mm}$ for Orion versus $3 \mathrm{~mm}$ for PentaRay and $4.75 \mathrm{~mm}$ for standard 3.5-mm tip mapping catheters) allows for recording higher bipolar voltage amplitudes ${ }^{22-32}$. Compared with Lasso, the mini-basket catheter has been reported to improve sensitivity in detecting PV potentials after $\mathrm{RF}$ ablation ${ }^{14,33}$. In selected cases of very diseased and scarred atria, low-voltage signals may not be seen with a standard cutoff sensitivity. The confidence mask is a complex multifactorial specific algorithm designed to improve scar identification within low-voltage areas. It uses a combination of an amplitude-based definition and consistency of the timing of local EGMs with those of the surrounding areas. The operator may have to increase the sensitivity (scar thresholding) to very low values, as low as $0.015 \mathrm{mV}$, to allow for distinguishing between low-voltage but viable conducting areas and dense scar tissues. Similarly, a decrease in sensitivity promotes scar exposure. The very low noise on the bipolar EGMs recorded with the basket catheter enables to set up a low scar threshold. Thus, the construction design of the mini-basket catheter seems to be unique. In theory, this could provide an increased mapping resolution. Additionally, owing to their exclusive location on the external spline side, the electrodes may be naturally less influenced by noise and far-field signals ${ }^{30-32}$ ). Both technologies have been developed on the basis of the high HD mapping theory. Although there is yet no consensus definition concerning HD/ultra-HD (UHD) cardiac mapping, electrophysiology practice has changed from low-definition (e.g., $<100$ points for cardiac chamber) to HD (e.g., >400 points) or UHD (e.g., >15,000 points). Recent reports of UHD mapping of organized arrhythmias yielded figures of 209 points/cm2 at the atrial level and 82 points/cm2 at the ventricular level. On top of the number of points and point density, HD/UHD mapping also enables improved substrate characterization, better understanding of the arrhythmia mechanism, and superior selection of ablation targets in atrial and ventricular arrhythmia cases ${ }^{32}$. Despite a theoretically higher number of automatically acquired points with the Orion catheter, along with a better signal/noise ratio, our study found that the new RM technology did 
not improve the AT RF acute success, in comparison with the new HD coloring technology.

Mapping and Ablation of AT. The effectiveness of tachycardia activation mapping using LAT has been proven. Published studies have documented variable success rates from $70 \%$ to $85 \%$ in AT termination when using this approach ${ }^{21}$. LAT mapping has seen recent advances, including automated annotation to the maximum negative unipolar EGM derivative within the bipolar activation period and its incorporation with high point density. However, most of these published studies have combined LAT with entrainment mapping in order to improve the efficacy of LAT mapping ${ }^{21}$. Furthermore, these studies did not report whether ablation had been delivered at multiple incorrect sites before eventual AT termination. A recently published study demonstrated acute AT termination with the first lesion set in $71 \%$ with LAT mapping but only $44 \%$ without entrainment ${ }^{21}$. The sources for error in relation to LAT mapping that could likely explain this [?] 30\% failure rate include: (1) incorrect color interpolation; (2) WOI errors; (3) mis-annotation, in addition to difficulties assessing automatic signal annotations. Interpolation algorithms assign the average activation time between mapped points to display an interpretable propagation pattern on the assumption that activation is uniform. These timing estimates can, however, be misleading, especially in areas of conduction delay $^{21}$. These occur at sites where early and late do not quite meet, with interpolation of colors between these apparent early and late sites, resulting in a slow-moving wave-front in reverse to the true activation direction. A WOI is required to ensure that EGM LATs from the same cycle are compared. Yet, this window process setting appears to be arbitrary, with different color-coded activation patterns generated depending on the setting. While methods to standardize this approach around the $P$ wave surface have been considered, they are only applicable in non-focal mechanisms, which are not yet known at the starting time ${ }^{20}$. Furthermore, in diseased tissues with prolonged conduction times, no matter how the window is set, being limited to a single cycle can lead to very late activating sites being erroneously displayed as early in the WOI with respect to the next activation cycle, resulting in more than one early site on the map ${ }^{30-32}$. Likewise, a WOI color wheel has been proposed to solve the challenges of WOI setting ${ }^{22-30}$. Rotation of this wheel has the equivalent effect of sliding the WOI without causing a full map re-computation. In principle, this could help avoid some errors related to the WOI as considered above. However, several studies have reported a high prevalence of small pseudo-reentrant circuits from continuously rotating the wheel, some of which were misdiagnosed as localized/small loop reentry and, thus, inappropriately ablated ${ }^{21}$. LAT mis-annotation can lead to a complete change in the color-coded pattern. The advent of high point density acquisition with algorithms that filter out points with inconsistent timings in relation to neighboring LAT measurements has markedly reduced such annotation errors. However, these errors remain prevalent, particularly in low voltage areas containing multicomponent EGMs ${ }^{30-32}$. Manual checking and LAT re-annotation when $>2000$ points are collected prove to be time-consuming and impractical during clinical procedures. Moreover, no consensus has been made on a voltage parameter able to differentiate between active and nonconducting tissue (i.e., either true scar from fibrosis or areas of functional block dependent on the wave-front direction and atrial rate) using endocardial mapping. LAT maps apply an arbitrary preset voltage threshold to display scarring and areas below this threshold as grey tags to blank the color-coded map ${ }^{21}$. All these limitations apply when LATs are mapped, and a better approach with these new technologies must be evaluated in order to improve LAT treatment efficacy. To our knowledge, only very few studies have compared the RM system incorporating the Orion catheter with the Carto system incorporating the PentaRay system ${ }^{26}$. In this later multicenter retrospective study, benefits of the RM system were evidenced ${ }^{26}$. Thus far, no prospective study has been published comparing HD coloring technology and RM technology. However, our preliminary results demonstrate that despite significantly more ultra-high electroanatomic point density (often in excess of 10,000 points per map), both technologies exhibit similar efficacy for AT treatment.

Clinical Implications. New technologies have been developed with the aim to improve AT diagnosis and treatment ${ }^{30-32}$. Both approaches share similarities, though they but differ from one another regarding the multicatheter type and computerized modelization system used. HD coloring (CARTO 3V6; Biosense Webster, CA) is a recently released software that provides a higher quality display of the electroanatomical map due to a higher density of points ${ }^{19,30,32}$. By this means, the user can set up two ranges of earlymeets-late: an upper range (the possible reentry zone) and lower one for a possible block mechanism- 
EEML. The EEML, which depends on the mapped tachycardia cycle, represents an improvement in the understanding of the tachycardia circuit and can prove particularly useful for verifying the presence of a block after an ablation line has been performed (Figure 1). However, the optimal cut-off ranges have not yet been defined. Along with the MEM technology, this system provides a higher-resolution display of electroanatomic maps and color visualization, simplifies the interpretation of arrhythmia mechanisms, and facilitates effective ablation ${ }^{19,21,30,32}$ ). The RM system is quite different in its concept (Figure 2). The latter facilitates the differentiation of active tissues from nonconductive ones within low-voltage areas, which is critical to studying macro-reentry and local reentry ${ }^{14,23,27-35}$. The WOI was represented as a color wheel running from red to purple while spanning the colors of the rainbow. These limits defaulted to red being the earliest in the window and purple the latest ${ }^{32}$. However, by representing the color bar as a wheel, purple conveyed activation $10 \mathrm{~ms}$ before red for reentrant maps. These limits could be rotated around the color wheel, and this had the equivalent effect of sliding the WOI without causing a full map recomputation. This function preserved the full color spectrum on the propagation map. Wavefront activation could be followed by rotating the color wheel and tracking the leading edge of a maroon-colored wavefront, distinguishable from the rainbow spectrum, around the cardiac chamber. Activation in extremely low voltage regions and inconsistent timing between neighboring points could be hidden from the map using a confidence mask. The default threshold of $0.03 \mathrm{mV}$ was employed, with areas below this confidence threshold displayed in gray and defined as scarring. Reducing this value below $<0.03 \mathrm{mV}$ reduced the gray zone size. The greater fidelity mapping offered by the Rhythmia system allowed us to identify active tissues within low voltage areas and to apply a confidence mask threshold (or scar threshold) of $0.03 \mathrm{mV}$. We were thus able to identify regions of possible scarring with amplitudes 5 to 10 times lower than those applied in previous studies about which local reentry could rotate ${ }^{32}$. Despite theorical technological mapping advantages, along with new computerized tachycardia modelization, our study demonstrates that the RM system is not superior to the HD coloring system regarding AT treatment efficacy. Moreover, the procedure parameters prove to be similar with respect to procedure duration and X-ray time exposure. Both technologies may be applied in the first-intent setting for AT treatment approaches with high efficacy levels.

Limitations . This study was not a multicenter trial, and our preliminary results involved only a limited patient cohort. In addition, the follow-up was entirely based on symptomatic recurrences and periodic Holter-ECG monitoring. No implantable loop recorder device was employed in the vast majority of the patients, with results not yet available in terms of recurrence. Approximately $37 \%$ of patients recruited were not included in the study analysis, owing to tachycardia termination before mapping was started, AFib changes, or LA thrombi. We excluded these patients because the ATs were not considered stable, and our endpoint of acute termination upon ablation would, thus, not have been robust. To define the best approach to achieve acute success and long-term benefit in this AT patient group, a higher number of patients must be included and further studies be performed.

Conclusions. This prospective monocenter randomized study demonstrates that the HD coloring and Rhythmia systems display a similar efficacy in achieving acute AT termination. Despite conceptually different approaches, both technologies appear to be equivalent in terms of efficacy and procedure parameter requirements.

\section{ABBREVIATIONS}

- AFib: atrial fibrillation

- AT: atrial tachycardia

- CI: critical isthmus

- CPVA: circumferential pulmonary vein ablation

- CS: coronary sinus

- CV: conduction velocities

- ECG: electrocardiogram

- EMG: electrogram

- EEML: extended early meets late 
- HD: high-definition

- LA: left atrium

- LAT: local activation time

- LVEF: left ventricular ejection fraction

- PVI: pulmonary vein isolation

- RA: right atrium

- RF: radiofrequency

- TCL: tachycardia cycle length

- WOI: window of interest

- X ray: radiography

- HD : confidense HD coloring mapping module

- RM: Rhythmia

- CI : critical isthmus

- $\mathrm{CV}$ : conduction velocities

- MEM : multielectrode mapping

- CFAE : complex fractionated atrial electrogram

- UHD: ultra-high-definition

\section{References}

1. Calkins H, Hindricks G, Cappato R, et al. 2017 HRS/EHRA/ECAS/APHRS/SOLAECE expert consensus statement on catheter and surgical ablation of atrial fibrillation. Heart Rhythm . 2017;14(10):e275-e444. doi:10.1016/j.hrthm.2017.05.012

2. Jais P, Shah DC, Haissaguerre M, et al. Mapping and Ablation of Left Atrial Flutters. Circulation . 2000;101(25):2928-2934. doi:10.1161/01.CIR.101.25.2928

3. Chae S, Oral H, Good E, et al. Atrial Tachycardia After Circumferential Pulmonary Vein Ablation of Atrial Fibrillation.Journal of the American College of Cardiology . 2007;50(18):1781-1787. doi:10.1016/j.jacc.2007.07.044

4. Nakagawa H, Shah N, Matsudaira K, et al. Characterization of reentrant circuit in macroreentrant right atrial tachycardia after surgical repair of congenital heart disease: isolated channels between scars allow "focal" ablation. Circulation . 2001;103(5):699-709. doi:10.1161/01.cir.103.5.699

5. Veenhuyzen GD, Knecht S, O'Neill MD, et al. Atrial Tachycardias Encountered during and after Catheter Ablation for Atrial Fibrillation: Part I: Classification, Incidence, Management. Pacing and Clinical Electrophysiology . 2009;32(3):393-398. doi:10.1111/j.1540-8159.2008.02250.x

6. Yokokawa M, Latchamsetty R, Ghanbari H, et al. Characteristics of atrial tachycardia due to small vs large reentrant circuits after ablation of persistent atrial fibrillation. Heart Rhythm . 2013;10(4):469-476. doi:10.1016/j.hrthm.2012.12.018

7. Wasmer K, Monnig G, Bittner A, et al. Incidence, characteristics, and outcome of left atrial tachycardias after circumferential antral ablation of atrial fibrillation. Heart Rhythm . 2012;9(10):1660-1666. doi:10.1016/j.hrthm.2012.06.007

8. Barbhaiya CR, Kumar S, Ng J, et al. Avoiding tachycardia alteration or termination during attempted entrainment mapping of atrial tachycardia related to atrial fibrillation ablation. Heart Rhythm . 2015;12(1):3235. doi:10.1016/j.hrthm.2014.09.002

9. Patel AM, d'Avila A, Neuzil P, et al. Atrial Tachycardia After Ablation of Persistent Atrial Fibrillation: Identification of the Critical Isthmus With a Combination of Multielectrode Activation Mapping and Targeted Entrainment Mapping. Circulation: Arrhythmia and Electrophysiology . 2008;1(1):14-22. doi:10.1161/CIRCEP.107.748160 
10. Jones DG, McCready JW, Kaba RA, et al. A multi-purpose spiral high-density mapping catheter: initial clinical experience in complex atrial arrhythmias. J Interv Card Electrophysiol . 2011;31(3):225-235. doi:10.1007/s10840-011-9574-0

11. Anter E, Tschabrunn CM, Josephson ME. High-Resolution Mapping of Scar-Related Atrial Arrhythmias Using Smaller Electrodes With Closer Interelectrode Spacing. Circ Arrhythm Electrophysiol . 2015;8(3):537545. doi:10.1161/CIRCEP.114.002737

12. Weiss R, Daoud EG. Can the Orion electrograms be the next shining star to help us navigate the pulmonary vein? Heart Rhythm . 2015;12(9):1935-1936. doi:10.1016/j.hrthm.2015.06.020

13. Nakagawa H, Ikeda A, Sharma T, Lazzara R, Jackman WM. Rapid High Resolution Electroanatomical Mapping: Evaluation of a New System in a Canine Atrial Linear Lesion Model. Circulation: Arrhythmia and Electrophysiology . 2012;5(2):417-424. doi:10.1161/CIRCEP.111.968602

14. Anter E, Tschabrunn CM, Contreras-Valdes FM, Li J, Josephson ME. Pulmonary vein isolation using the Rhythmia mapping system: Verification of intracardiac signals using the Orion mini-basket catheter.Heart Rhythm . 2015;12(9):1927-1934. doi:10.1016/j.hrthm.2015.05.019

15. Schaeffer B, Hoffmann BA, Meyer C, et al. Characterization, Mapping, and Ablation of Complex Atrial Tachycardia: Initial Experience With a Novel Method of Ultra High-Density 3D Mapping. J Cardiovasc Electrophysiol . 2016;27(10):1139-1150. doi:10.1111/jce.13035

16. Latcu DG, Saoudi N. How fast does the electrical impulse travel within the myocardium? The need for a new clinical electrophysiology tool: the conduction velocity mapping. J Cardiovasc Electrophysiol . 2014;25(4):395-397. doi:10.1111/jce.12350

17. Anter E, McElderry TH, Contreras-Valdes FM, et al. Evaluation of a novel high-resolution mapping technology for ablation of recurrent scar-related atrial tachycardias. Heart Rhythm . 2016;13(10):2048-2055. doi:10.1016/j.hrthm.2016.05.029

18. Laţcu DG, Bun S-S, Viera F, et al. Selection of Critical Isthmus in Scar-Related Atrial Tachycardia Using a New Automated Ultrahigh Resolution Mapping System. Circulation: Arrhythmia and Electrophysiology . 2017;10(1). doi:10.1161/CIRCEP.116.004510

19. Asferg C, Chen X, Pehrson S, Jacobsen PK. Catheter ablation of atypical flutter using new 3-dimensional electroanatomic mapping software focusing on areas of conduction block. HeartRhythm Case Reports . 2019;5(4):225-228. doi:10.1016/j.hrcr.2019.01.003

20. De Ponti R, Verlato R, Bertaglia E, et al. Treatment of macro-re-entrant atrial tachycardia based on electroanatomic mapping: identification and ablation of the mid-diastolic isthmus. EP Europace . 2007;9(7):449457. doi:10.1093/europace/eum055

21. Luther V, Agarwal S, Chow A, et al. Ripple-AT Study. Circ Arrhythm Electrophysiol . 2019;12(8):e007394. doi:10.1161/CIRCEP.118.007394

22. Tschabrunn CM, Roujol S, Dorman NC, Nezafat R, Josephson ME, Anter E. High-Resolution Mapping of Ventricular Scar: Comparison Between Single and Multielectrode Catheters. Circ Arrhythm Electrophysiol . 2016;9(6). doi:10.1161/CIRCEP.115.003841

23. Takigawa M, Denis A, Frontera A, et al. Triple-loop reentrant atrial tachycardia originated after pulmonary vein isolation. J Interv Card Electrophysiol . 2017;48(3):367-368. doi:10.1007/s10840-016-0187-5

24. Takigawa M, Frontera A, Martin R, Jais P, Haïssaguerre M, Sacher F. Dual loop reentrant tachycardia with a combination of a localized reentry and a macro-reentry. J Cardiol Cases . 2017;15(6):197-200. doi:10.1016/j.jccase.2017.02.006

25. Berte B, Relan J, Sacher F, et al. Impact of Electrode Type on Mapping of Scar-Related VT: Mapping of Scar-Related VT. Journal of Cardiovascular Electrophysiology . 2015;26(11):1213-1223. doi:10.1111/jce.12761 
26. Maury P, Champ-Rigot L, Rollin A, et al. Comparison between novel and standard high-density 3D electro-anatomical mapping systems for ablation of atrial tachycardia. Heart and Vessels . November 2018. doi:10.1007/s00380-018-1307-1

27. Takigawa M, Derval N, Maury P, et al. Comprehensive Multicenter Study of the Common Isthmus in Post-Atrial Fibrillation Ablation Multiple-Loop Atrial Tachycardia. Circulation: Arrhythmia and Electrophysiology . 2018;11(6). doi:10.1161/CIRCEP.117.006019

28. Luther V, Sikkel M, Bennett N, et al. Visualizing Localized Reentry With Ultra-High Density Mapping in Iatrogenic Atrial Tachycardia: Beware Pseudo-Reentry. Circulation: Arrhythmia and Electrophysiology . 2017;10(4). doi:10.1161/CIRCEP.116.004724

29. Pathik B, Kalman JM. Perceiving the Imperceptible in Atrial Macro-Reentry: Ultrahigh Resolution Mapping to Characterize the Critical Isthmus. Circulation: Arrhythmia and Electrophysiology . 2017;10(1). doi:10.1161/CIRCEP.116.004850

30. Scaglione M, Caponi D, Di Donna P, Battaglia A. Ultra-high definition mapping to choose the optimal ablation strategy in atrial macro-re-entrant tachycardia. EP Europace . 2019;21(Supplement_3):iii13-iii14. doi:10.1093/europace/euz149

31. Borlich M, Sommer P. Cardiac Mapping Systems: Rhythmia, Topera, EnSite Precision, and CARTO. Card Electrophysiol Clin . 2019;11(3):449-458. doi:10.1016/j.ccep.2019.05.006

32. Laţcu DG, Saoudi N. High-resolution/Density Mapping in Patients with Atrial and Ventricular Arrhythmias. Card Electrophysiol Clin . 2019;11(3):511-524. doi:10.1016/j.ccep.2019.05.001

33. Segerson NM, Lynch B, Mozes J, et al. High-density mapping and ablation of concealed low-voltage activity within pulmonary vein antra results in improved freedom from atrial fibrillation compared to pulmonary vein isolation alone. Heart Rhythm . 2018;15(8):1158-1164. doi:10.1016/j.hrthm.2018.04.035

34. Schaeffer B, Akbulak RÖ, Jularic M, et al. High-Density Mapping and Ablation of Primary Nonfocal Left Atrial Tachycardia. JACC: Clinical Electrophysiology . 2019;5(4):417-426. doi:10.1016/j.jacep.2019.02.002

35. Takigawa M, Martin CA, Derval N, et al. Insights from atrial surface activation throughout atrial tachycardia cycle length: A new mapping tool. Heart Rhythm . April 2019. doi:10.1016/j.hrthm.2019.04.029

Table 1. Population Characteristics

\begin{tabular}{|c|c|c|c|c|}
\hline Variable & $\begin{array}{l}\text { Study Population } \\
(\mathrm{n}=38)\end{array}$ & $\begin{array}{l}\text { HD Coloring } \\
\text { System }(n=20)\end{array}$ & $\begin{array}{l}\text { Rhythmia } \\
\text { System }(n=18)\end{array}$ & $\mathbf{p}$ \\
\hline$\overline{\text { Age - mean } \pm \text { SD }}$ & $66 \pm 9$ & $66 \pm 10$ & $66 \pm 9$ & 0.9 \\
\hline $\begin{array}{l}\text { Gender - n (\% } \\
\text { female) }\end{array}$ & $12 / 38(31.5 \%)$ & $4 / 20(20 \%)$ & $8 / 20(40 \%)$ & 0.1 \\
\hline $\begin{array}{l}\text { Hypertension - } \mathrm{n} \\
(\%)\end{array}$ & $19 / 38(50 \%)$ & $10 / 20(50 \%)$ & $9 / 18(50 \%)$ & 0.9 \\
\hline $\begin{array}{l}\text { Diabetes mellitus } \\
-\mathrm{n}(\%)\end{array}$ & $5 / 38(13.2 \%)$ & $3 / 20$ & $2 / 18$ & 0.7 \\
\hline $\begin{array}{l}\text { CHA }^{2} \mathrm{DS}^{2} \text { VASc } \\
\text { score- n }(\%)\end{array}$ & $2.3 \pm 1.5$ & $2.4 \pm 1.6$ & $2.2 \pm 1.4$ & 0.6 \\
\hline $\begin{array}{l}\text { Cardiomyopathy - } \\
\mathrm{n}(\%)\end{array}$ & $14 / 38(37 \%)$ & $8 / 20(40 \%)$ & $6 / 18(33.3 \%)$ & 0.7 \\
\hline $\begin{array}{l}\text { Body mass index } \\
-(\mathrm{cm} 2)\end{array}$ & $26 \pm 5$ & $28 \pm 4$ & $27.5 \pm 4$ & 0.85 \\
\hline $\begin{array}{l}\text { Previous AFib } \\
\text { ablation- n (\%) }\end{array}$ & $24 / 38(63.2 \%)$ & $11 / 20(55 \%)$ & $13 / 18(72 \%)$ & 0.3 \\
\hline
\end{tabular}




\begin{tabular}{lllll}
\hline Variable & $\begin{array}{l}\text { Study Population } \\
(\mathbf{n = 3 8})\end{array}$ & $\begin{array}{l}\text { HD Coloring } \\
\text { System }(\mathbf{n = 2 0})\end{array}$ & $\begin{array}{l}\text { Rhythmia } \\
\text { System }(\mathbf{n}=\mathbf{1 8})\end{array}$ & $\mathbf{p}$ \\
\hline $\begin{array}{l}\text { Ischemic } \\
\text { cardiomyopathy - }\end{array}$ & $3 / 38(7.9 \%)$ & $2 / 20(10 \%)$ & $1 / 18(5.5 \%)$ & 0.35 \\
$\begin{array}{l}\text { Left atrium } \\
\text { volume }-\mathrm{mL}\end{array}$ & $59 \pm 32$ & $64 \pm 35$ & $52 \pm 30$ & \\
LVEF - mean $\pm \mathrm{SD}$ & $56 \pm 12$ & $61 \pm 15$ & $56 \pm 15$ & 0.3 \\
\hline
\end{tabular}

Values are expressed as mean \pm SD or number (\%); LVEF: left ventricular ejection fraction

AFib: atrial fibrillation; LVEF: left ventricular ejection fraction

Table 2. Baseline Procedure Details

\begin{tabular}{llll}
\hline Baseline Characteristics & Assigned Group & Assigned Group & $P$ Value \\
& HD & RM & \\
No. of patients & 20 & 18 & \\
Prior atrial ablation (\%) & $8(40 \%)$ & $11(61 \%)$ & 0.5 \\
Prior cardiac surgery without ablation (\%) & $3 / 20(15 \%)$ & $2 / 18(11 \%)$ & 0.8 \\
Prior atrial ablation (\%; LA) & $11 / 20(55 \%)$ & $13 / 18(72 \%)$ & 0.3 \\
Prior PVI (\%) & $9 / 20(45 \%)$ & $11 / 18(61 \%)$ & 0.8 \\
Prior LA substrate ablation (eg, lines and CFAE; \%) & $2 / 20(10 \%)$ & $2(11.1 \%)$ & 0.9 \\
Prior LA substrate ablation (eg, lines; \%) & $4 / 20(20 \%)$ & $5(28 \%)$ & 0.8 \\
Prior LA substrate ablation (eg, CFAE; \%) & $7 / 20(35 \%)$ & $8(44.4 \%)$ & 0.8 \\
Prior RA ablation only (\%) & $8 / 20(40 \%)$ & $5(28 \%)$ & 0.4 \\
AT cycle length/ms, median & $275 \pm 30$ & $280 \pm 50$ & 0.7 \\
Procedure time (skin to skin, min) & $138 \pm 90$ & $139 \pm 90$ & 0.98 \\
Total X ray (min) & $20 \pm 11$ & $23.8 \pm 18$ & 0.42 \\
Number of activation map per patient & $2.1 \pm 1$ & $2 \pm 1$ & 0.9 \\
Collected Points, median & $6370 \pm 3111$ & $27870 \pm 2034$ & $<.0001$ \\
Mean number of circuits per patient & $1.8 \pm 1$ & $1.6 \pm 0.9$ & 0.6 \\
RF application time & $39 \pm 36$ & $25 \pm 28$ & 0.21 \\
Acute success & $15 / 20(75 \%)$ & $14 / 18(78 \%)$ & 0.94 \\
Incomplete success & $3 / 20(15 \%)$ & $3 / 18(16.5 \%)$ & 0.9 \\
Failure & $1 / 20(10 \%)$ & $1 / 18(5.5 \%)$ & 0.9 \\
\hline
\end{tabular}

AT, atrial tachycardia; CFAE, complex fractionated atrial electrogram; LA, left atrium; LAT, local activation time; PVI, pulmonary vein isolation; RA, right atrium; RF: radiofrequency; X-ray: radiography; ablation-induced transformation to another AT was presumed if a sustained change in cycle length ([?]20 $\mathrm{ms}$ ), intracardiac activation pattern (on the coronary sinuscatheter), or surface ECG modifications occurred

Table 3. Arrhythmia Characteristics in HD group

\begin{tabular}{lllll}
\hline Patient & TCL $(\mathbf{m s})$ & Mechanism & Isthmus/Focus location & Ablation successfull \\
\hline 1 & $246 / 280$ & MCR/2 single loops & MI/Roof & yes \\
2 & 250 & MiCR/single loop & Antero-septal & yes \\
3 & 267 & MCR/single loop & MI & yes \\
4 & 305 & MCR/single loop & Right atrium (atriotomy cicatrice) & yes \\
5 & $235 / 267$ & MCR/2 single loops & Roof/MI & Not complete
\end{tabular}




\begin{tabular}{lllll}
\hline Patient & TCL $(\mathbf{m s})$ & Mechanism & Isthmus/Focus location & Ablation successfull \\
\hline 6 & 245 & MCR/dual loops & MI+Roof & Not complete \\
7 & 290 & MCR/single loop & Anterior (atriotomy cicatrice) & yes \\
8 & 280 & Focal & Septum & yes \\
9 & 305 & MCR/single loop & Anterior & yes \\
10 & 270 & MCR/single loop & Anterior & yes \\
11 & $250 / 270$ & MCR/2 single loops & MI/Right pulmonary veins & Not complete \\
12 & $240 / 340$ & MCR/1 single loop an 1dual loops & MI/Roof+anterior & yes \\
13 & 290 & MCR/single loop & Septal & Pericardial effusion \\
14 & 255 & MCR/single loop & MI & yes \\
15 & 330 & MiCR & Left superior pulmonary vein & yes \\
17 & 275 & MCR/single loop & Ridge & no \\
18 & 275 & MCR/single loop & MI & yes \\
19 & 250 & MCR/dual loops & MI+Roof & yes \\
20 & 320 & MCR/dual loops & MI+Roof & yes \\
\hline
\end{tabular}

$\mathrm{MCR}=$ Macro-reentrant; MiCR $=$ Micro-reentrant; $\mathrm{MI}=$ Mitral isthmus; $\mathrm{PV}=$ Pulmonary veins

Table 4. Arrhythmia Characteristics in RH group

\begin{tabular}{lllll}
\hline Patient & TCL $(\mathbf{m s})$ & Mechanism & Isthmus/Focus location & Ablation successf \\
\hline 1 & $248 / 320$ & MCR/2 single loops & Septal-inferior/MI & yes \\
2 & 330 & MiCR single loop & Antérior & yes \\
3 & 330 & MCR single loop & Cavotricuspid isthmus & yes \\
4 & $290 / 300$ & Focal & Right anterior/coronary sinus ostium & yes \\
5 & $197 / 220 / 245$ & MCR/3 single loops & Antero-septal/roof/septal & yes \\
6 & $245 / 300$ & MCR/2 single loops & Septal/ Cavotricuspid isthmus & yes \\
7 & 328 & MCR/Single loop & Cavotricuspid isthmus & yes \\
8 & $211 / 231$ & MCR/dual loops and 1 single loop & MI+PV dependent/Roof & Not complete \\
9 & 233 & MCR/single loop & MI & yes \\
10 & 290 & MCR/single loop & Cavotricuspid isthmus & yes \\
11 & 253 & MCR/single loop & MI & yes \\
12 & $240 / 260$ & MCR/2 single loops & MI/Anterior & Not complete \\
13 & 377 & MCR single loop & Anterior & yes \\
14 & 330 & Focal & Anterior & yes \\
15 & 260 & MCR/dual loops & MI+Roof & yes \\
17 & 258 & MCR dual loops & MI+Left appendage & yes \\
18 & 260 & MCR single loop & Septal-inferior & Not complete \\
\hline
\end{tabular}

$\mathrm{MCR}=$ Macro-reentrant; MiCR $=$ Micro-reentrant $\mathrm{MI}=$ Mitral isthmus $\mathrm{PV}=$ Pulmonary veins 

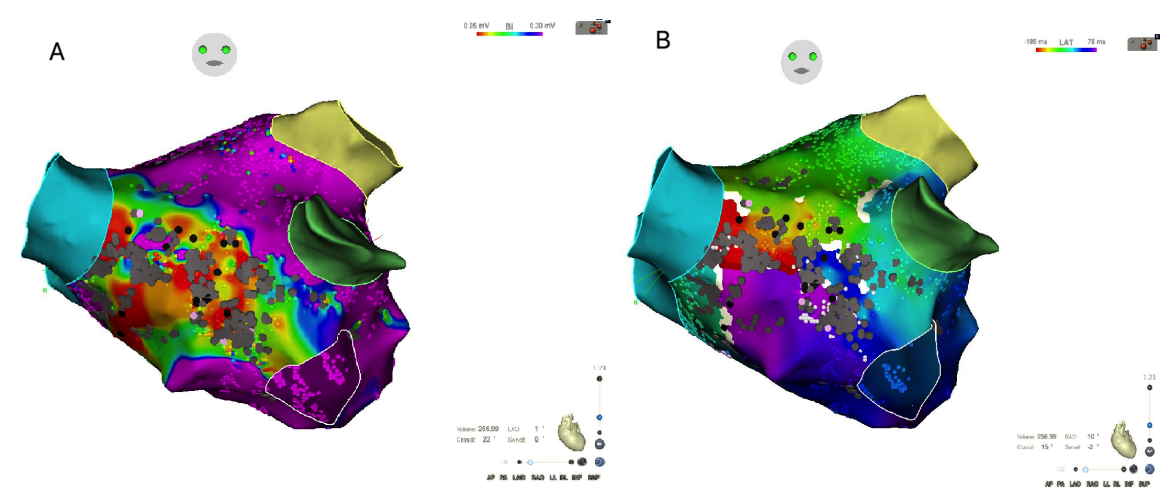

Figure 1. Electroanatomic mapping of the left atrium performed with the PENTARAY catheter (Biosense Webster, Diamond Bar, CA) with CARTO 3 high definition (HD) coloring feature (Biosense Webster).A . The bipolar voltage map (lower threshold: $0.05 \mathrm{mV}$, upper threshold: $0.3 \mathrm{mV}$ ) shows that regions with scar tissue correlate with the areas of conduction block in the LAT map. Moreover, the region of healthy tissue between scarring areas correlates with normal conduction velocites in the LAT map. B . Local activation time (LAT) map showing conduction block (withe line) due to atriotomy scarring post mitral valve repair and reentry path (red line), conduction gap into the atriotomy line. C. The propagating wavefront passes through the narrow path between the 2 regions of scarring tissue with low conduction velocities $(\mathrm{CV})$, this aera can be defined as the critical isthmus of the macro-reentry circuit (See video). Finally, we can see another conduction gap though atriotomy line, close to the left appendage small reentry circuit and extremely slow conduction velocites. The radiofrequency (RF) ablation in this area completing the atriotomy line allowed the sinus rhythm recovery.

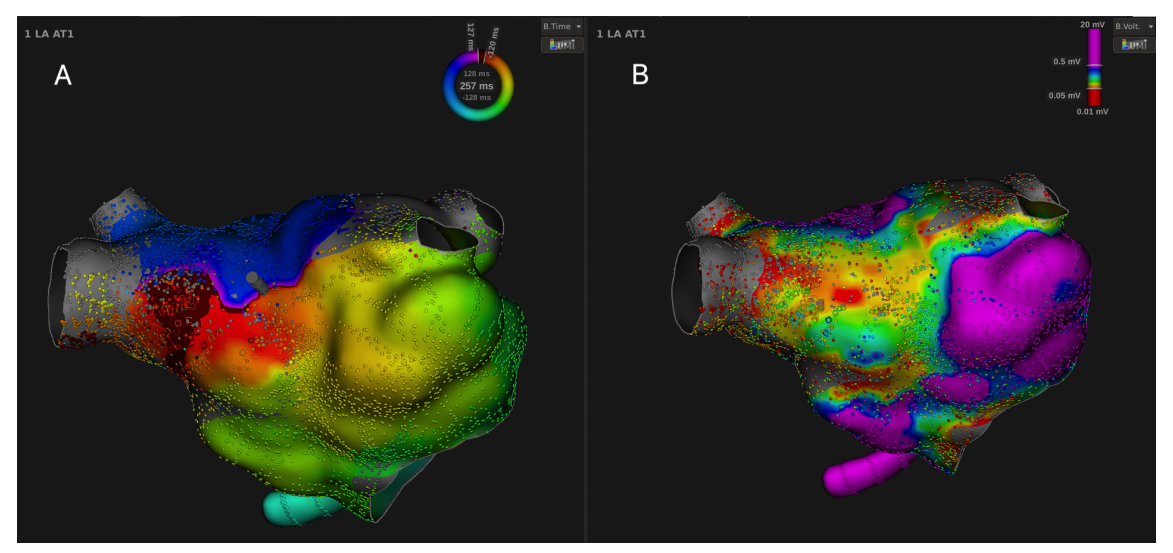




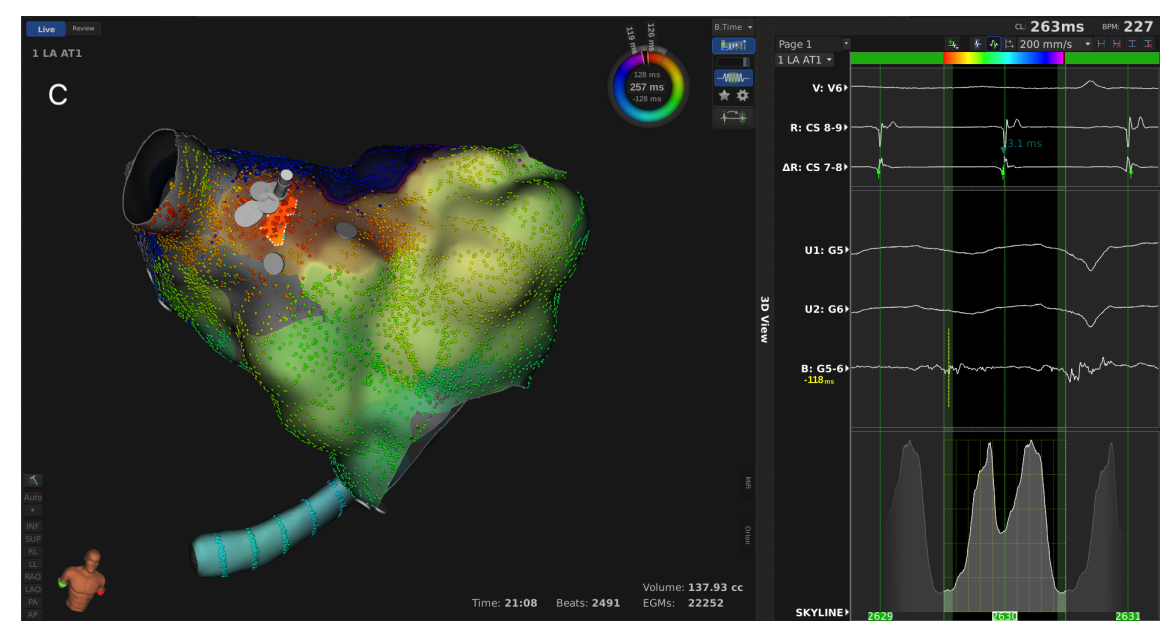

Figure 2. Electroanatomic mapping of the left atrium performed with the IntellaMap Orion catheter (Boston Scientific) with RHYTHMIA system. A. Local activation time (LAT) map showing a roof-dependent tachycardia with narrow reentry path into large low conduction velocities area due to radiofrequency of complex fractionated atrial electrogram (CFAE) during the first AF ablation procedure. The propagation map shows clearly a low conduction velocities and narrow pathway close to the right pulmonary vein between a scarring tissue, this area can be defined as a critical isthmus (CI) of the macro-reentry circuit. B. The bipolar voltage map (lower threshold: $0.05 \mathrm{mV}$, upper threshold: $0.5 \mathrm{mV}$ ) shows large low voltage area correlate with slow conduction velocities between lefts and rights pulmonary veins.C. LAT map with activation of the Lumipoint module for detection of fragmented potentials, localize a narrow fragmented aera corresponding to critical isthmus (CI) detected with the LAT map. The radiofrequency $(\mathrm{RF})$ ablation in this area completed the roof line and the sinus rhythm was restored. 